\title{
The Application of Taoism Culture in the Sichuan Bonsai
}

\author{
Jiaqi Luo \\ College of Landscape Architecture \\ Sichuan Agricultural University \\ Chengdu, China
}

\author{
Yufan Ding \\ College of Landscape Architecture \\ Sichuan Agricultural University \\ Chengdu, China \\ Kezhu Lu \\ College of Landscape Architecture \\ Sichuan Agricultural University \\ Chengdu, China
}

\author{
Xiaoyu Ming \\ College of Landscape Architecture \\ Sichuan Agricultural University \\ Chengdu, China \\ Xiaofang $\mathrm{Yu}^{*}$ \\ College of Landscape Architecture \\ Sichuan Agricultural University \\ Chengdu, China \\ *Corresponding Author
}

\begin{abstract}
Taoism has gone through more than three thousand years of history, its ideological accomplishments penetrate many aspects of Chinese aesthetics. Since Taoism Developed in Sichuan at the end of the eastern Han dynasty, it plays a great part in the modeling, production and artistic aesthetics of Sichuan Bonsai. The article analyzes and explores the ideology of Taoism through literature research and case analysis in the directive significance of Sichuan-Bonsai creation, which absorbing the natural landscape, creating space layout and expressing the artistic conception of "Sheng".
\end{abstract}

Keywords-Taoism; Sichuan Bonsai; nature; artistic conception

\section{INTRODUCTION}

Sichuan plays an important role in the history of the development of Taoism. There were not only the cultural traditions of people's superstition, but also the deep and natural landscape ${ }^{[1]}$. Since Zhang Ling settled down in Sichuan and went to Heming Mountain to establish Fivedou-grain Taoism (later called the Way of the Celestial Masters) at the end of the eastern Han dynasty, the Taoism activities developed strongly in Sichuan. Many kinds of Taoist genres established and formed an integrated system. So as to say Sichuan is the birthplace and headstream of Taoism. The Taoism activities in the past two thousand years have also left a rich legacy of Taoism culture, covering many aspects such as literature, art, architecture, morality and so on. Rich landscape resources and plant materials provide unique conditions for the development of Sichuan Bonsai, which is the valuable heritage inherited from history. The profound cultural inheritance also forms a relatively unique bonsai modeling features and aesthetic standard ${ }^{[2]}$.

Taoism not only influenced the thought of different dynasties, but also the mainstream concept of aesthetic art and the idea of development in bonsai art. The article will discuss the influence and the application of Taoism culture reflecting on the theme, artistic conception, aesthetic standard and other aspects of Sichuan Bonsai.

\section{THE ORIGIN AND SUMMARIZE OF TAOISM}

\section{A. The Origin of Taoism}

Taoism is one of the main religions in China, and is the only native culture of China.

During the reign of emperor Xuanyuan, on account of the science technology and productivity in old days, people's attitude towards nature are mainly admirable and awestruck, which results in the superstition that human believe that al the things and aftermath happened in the earth are owing to God's spirits. This primitive religion is the beginning of Taoism. With the reinforcement of the idea of superstition, the Huang's conception prevails during the early Qin dynasty and the Warring States period. The spread of The Huang's conception during the early Qin dynasty in Sichuan and its native superstition provide conditions for the Taoism from coming to being. As a result, based on the Taoist philosophy of Chuang Tzu and Lao Tzu, the concept of "Tao" was gradually formed in this period. Both of them take "Tao" as the highest form of ideology, which provided Taoism the basis and source of thought. During the late eastern Han dynasty, politics was at low ebb, people were eager for 
spiritual brace. Therefore, Taoism arises at the moment. Zhang Ling founded the Five-dou-grain Taoism (later called the Way of the Celestial Masters) in mount heming and mount qingcheng. He has also set up 24-prefectures in every county. Eventually, Taoism developed formally in Sichuan. Zhang Ling later perfected its system and founded Taoism ceremony, standard, etc. Then the Way of Celestial Masters in the eastern Han dynasty is defined as the formal start of Taoism. In the past dynasties, the specific historical circumstances and specific historical problems have gradually become mature and formed a unique Chinese religion.

It can be said that Taoism was originated from the emperor Huang, originated from Lao Tzu, and established by Zhang Ling.

\section{B. The Summary of Taoism Ideology}

Taoism takes the ideologies of Philosophical Taoism as the mental mainstream, at the same time absorbs the ancient Chinese witchcraft and the art of necromancy, as a result forming its unique ideological system[3]. The basis of Taoist ideas was established in the Spring and Autumn Period, deriving a series of religious, natural, moral and aesthetic concepts.

Lao Tzu said "Man follows the earth. Earth follows the universe. The universe follows the Tao. The Tao follows only itself." The "Nature" not only means the nature, but also points to the objective law of the development of everything, which including dialectical thinking, advocating governing without doing anything and conforming to the Tao. It also advocates advocating natural and living in harmony with it, possessing the strong naturalism. The "reclusion" conception of life-withdraw from the society, living in mountains and truly understanding the naturalism is reflected in the Wei and Jin Dynasties.

During the warring States period, Chuang Tzu inherited and carried forward the ideology of Lao Tzu and regarded Tao as the origin of all things in the universe, which is interconnected with the source of universe. He advocates" the harmony between human and nature" and combines his view of life with the universe, as a result achieving his spiritual shackles - The nature lives with me in symbiosis, and everything is with me as a whole, which embodies Chuang Tzu's philosophy of life.

\section{THE ORIGIN AND SUMMARY OF BONSAI}

\section{A. The History and Classification of Bonsai}

Bonsai, originated in China, is a kind of natural landscape art with plants and rocks as its basic materials. Having experienced the key period of the Han dynasty, bonsai achieved the transformation from original potted plants to artistic potted landscapes. Then in the prosperity of Tang and Song dynasties, people added poetic nature into it, making it a leap of artistic conception. In the subsequent dynasties, they integrated different regional cultures according to the politics and economy of the current dynasties and developed towards a more mature and perfect direction.

Because of life experience, the different aesthetic preferences and interest as well as geography and climate, potted-landscape culture from different places is colorful. Under the condition of the different traits, the style of bonsai has inherited from generation to generation. Then it tends to be stable and recognized, as a result forming different school of art, mainly divided into eight major schools: Sue, Yang, Lingnan, Sichuan, Hui, Shanghai, Jiang and Tong.

\section{B. The Summary of Sichuan Bonsai}

Sichuan bonsai mainly indicates the bonsai in Sichuan and Chongqing, and partly the surrounding counties and cities, usually calls the bonsai in Chengdu as the western Sichuan bonsai, while the bonsai in Chongqing calls the eastern Sichuan bonsai. The western Sichuan bonsai pursues the poetic imagery, which is elegant and pretty, and strives to achieve the "Being artificial by human is equal to a natural wonder." In its means of artistic expression, the western Sichuan bonsai imitates nature spirits by applying the tact of "a small sign can indicate a great trend" to construct the potted landscape. The western Sichuan bonsai focuses on realism and naturalness and pays attention to the local magnificent mountain.

According to the materials in modeling, Sichuan bonsai can be divided into two categories. A kind of plants bonsai is the main modeling. The other kind is the landscape-bonsai with the mountain stone or the substitution of mountain stone. The style of plants-bonsai is natural, curving and of primitive simplicity, while the landscape-bonsai is vivid and splendid, showing the great scenery of Emei, Jianmen and Qingcheng [4]. Landscape-bonsai is able to show the natural scenery of Sichuan authentically, which is green, quiet, dangerous and splendid.

\section{THE APPLICATION OF TAOISM CULTURE IN THE SICHUAN BONSAI}

Sichuan bonsai has a long history. In the process of artistic creation, the creators will integrate their own interests and pay attention to the idea and painting environment. Transform it on a stone or a wood, making it become the ideal production. Taoism philosophy is extensive and profound, which influent greatly on the whole history and culture of aesthetic standard and the skill creating art in China.

\section{A. The "Tao" of Nature}

Taoism has always been highly praised for the beauty of nature. Lao Tzu, the core character, also proposed the philosophy of "Tao models itself after nature", which suggesting people imitating nature and learning the laws of nature such as landscapes, trees, grass and wilderness. It leads people to explore the beauty of the universe and the Tao. This kind of "natural beauty" has an extremely wide range influencing on all kinds of art in Chinese poetry and landscape painting. 
Taoism has always talks about "restoring the beauty of the original universe and reaching the principle of all", and even regards the "natural beauty" as the environmental conditions and requirements for cultivation. The "coming out" side of the religious ideologies of Taoism has the intensively contact with the praise for the beauty of "mountain forest", which always appeals to concealing in the forest, and has a particularly preference for mountain and fountain[5]. This kind of pursuing the beauty of the original universe is the Taoists' self-exploration of their inner world.

Taoism advocates the aesthetic idea "manifest plainness, embrace simplicity". Lao Tzu said: "the five colors make our eyes blind." Chuang Tzu said: "the five colors disturb our eyes and make our eyes vague." Taoists thought colors confuse the eyes of people and disturb our judgment with surface phenomena, and we couldn't feel the natural beauty of worldly things. As a result requiring people to hold on to the essence of things, do not be confused with society, and pursue the original natural beauty of things.

Sichuan Bonsai prefers the ancient, peculiar shape and natural Bonsai which often matched with stones and the having characteristics of the scenery in Sichuan. People can experience the beauty of mountains and rivers in Sichuan through the "landscape in the pot" and appreciate the uncanny workmanship of nature. It is also known as the "silent poems, dimensional paintings, and lively sculptures". The natural manner can be expressed in the following wordsQuiet, Pretty, Dangerous, Majestic, High, Deep, Steep and Sharp. The "Quiet", "Pretty", "Dangerous", "Majestic" are the concrete reflections of Sichuan Bonsai and the rest indicate unique techniques in its modeling [6]. "High" refers to an unattainable mountain holding all mountains in a single glance; "Deep" refers to the profound artistic conception; "Steep" and "Hanging" refer to precipitous peaks and cliffs which cannot be ventured just as it described in The Sichuan Road What heights, " Peaks short of touching the sky by a mere foot join to form mountain ranges, and off abrupt and sheer precipices hang withered pines. Waterfalls and white water race to uproar create, splashing on rocks in thousands of valleys to thunders aggregate. "

The philosophy of Taoism is based on the reflection of the essence of things, which advocating people to reach the sublime understanding of nature by pursuing and imitating the nature. The Sichuan Bonsai is the model of nature and rolls the natural beauty and artistic beauty into one. It gives people a daydream artistic conception which undoubtedly embodying the "natural beauty" that Taoism respects.

\section{B. The Beauty of Landscape}

Influenced by the conception of nature, Taoism's understanding of landscape is not only the geographical concept of natural objects themselves, but also contains places and symbols that pursue a higher status of immortal [7]. The ideologies of Chuang Tzu and Lao Tzu both originate from nature and put their heart and soul in it. By relaxing in landscape, they can experience the abundant, interesting way of nature and understand the "Tao" inside it.
The mountains in nature are often simulated by people in the way of shrinkage and migration. Rocks are the product of year deposit, containing a strong emotion in nature, rivers, soil, or the condensation in the lava. Each individual is unique and beautiful after interchange. Chen congzhou said in Shuo Yuan (5) that "the stone is delicate, the stone is strong, the stone is simple and its shape is dull". The "dull" in it is not clumsy, but refers to its original initial state, which is, a kind of natural undecorated rustic beauty. This is identified with Taoism's inherent beauty of embracing simplicity. Water is closely related to us. In addition to the material necessities, people usually sublimate it to the spiritual level. Lao Tzu said "Be kind as water, and water benefit everything to lie itself. It is in all the people's evil, as in closest the way to Tao. ", which shows the highest state of good like the water being surrounded by all things and abandoning the fame and wealth. The "Tao" in the universe is just like this, which proposes that we do not need to seek to make choices, but pursues simplicity and govern without intervention.

The landscape bonsai in the potted landscape of Sichuan is exactly the reproduction of the natural landscape. A few feet inside and the basin stands at a thousand [8]. People choose stones containing natural beauty and with the understanding of nature and the collocation of water and plants through different compositions, showing the lofty artistic conception of Sichuan bonsai. Such as the "quiet, beautiful and fantastic" in mount qingcheng, it has a lot of artistic interpretation in the Sichuan potted landscape. The peaceful mountains, clouds and the amusement palace hidden in, with the subtle and gentle water reflected in the spiritual of Lao Tzu "The water benefits everything to lie itself ". The posture of mountain is changeful, sometimes in an unbroken line showing its beautiful appearance and sometimes the ravines are deep and treacherous. Viewing the landscape bonsai can also generate the cognitive space. Varying scenery with changing view-points also come into different artistic conceptions, such as the interest of " It's like a range when you look at the mountain from the front. But it's like a peak when you look at it sideways", which creating unlimited poetic connotation.

The intention of Taoism and Sichuan bonsai in the natural landscape is not only confined to the real scene commentary and reappearance, but also the sublimation in spirit level. Sichuan bonsai uses the scenery of mountains and rivers affected by Taoism for reference and refine through sublimation, both of them have the same effect.

\section{The Spatial Arrangement}

Taoist culture pays attention to exploring the nature of the universe. The Tao Teh King said, "One is the child of the Tao. After one come two, two come three and three come all things. All things negative Yin and embrace Yang, combine two as to get harmony. ", which expresses the ancients' understanding of the original universe. The "Yin" and "Yang" can be objectively understood as things from two kinds of substance. They are both opposite and unified, and exist in the interior of things as well as outside things. From the view of aesthetics, "Yin" and "Yang" affects each other, 
producing unparalleled beauty of form and artistic conception.

Lao Tzu said that "Everything in the world comes for being, and being comes from nothing." Everything is generated by "being", at the same time "being" is generated from "nothing". "Nothing" is not "inexistence" and "empty", it is the intangible things that could produce the "nothing" in visible of all things. "Nothing" is equal to "Tao", and "Tao" is the unification of the "being" and "nothing", which can also be understood as the unification of the "real" and "virtual" [9]. The theoretical elaboration of posture and momentum which are closely related to "Tao" are also the important basis theory of "image" in aesthetics.

Sichuan bonsai pays attention to the shape of form and the potential aesthetic in the artistic expression. The form means the shape of natural landscape, the potential means the "posture" and "momentum". The "posture" prefers "modelling posture", which can be the purifying and crystallization of landscape bonsai. Through the basic principles of different modelling, people create visual impression which is not constrained by physical modelling. The "momentum" more emphasizes the mobile state of mind, making the stewing bonsai become vivid while enjoy the sight of it. Therefore, the "momentum" can also be called the "dynamic momentum".

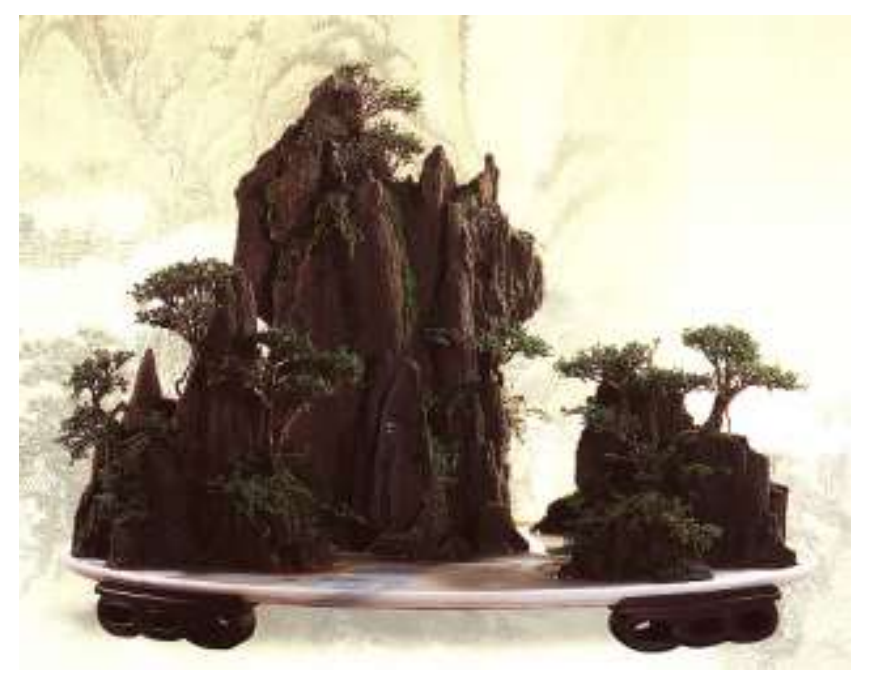

Fig. 1. The Mountains In Sichuan Reflected the Green Water

In the momentum of Sichuan bonsai, some aesthetic principles will be adopted. People fuse the ideology of Tao in the conception in bonsai design, and using the dynamic balance between Yin and Yang in its layout. The use of contrast and unity as to achieve coordination, modelling of the actual situation is characterized by "appear" and "hidden", which using rock and plant landscape as a barrier and giving people a winding path leads to a secluded spot with reflection of vivid, clever "Tao" ${ }^{[10] .}$ Even in the management of "being" and "not being", people take extreme white space and make bonsai have the power and the trend in movement, making whole space artistic conception hollow and vivid again. Such as the Sichuan landscape bonsai produced by Yang Tao -The Mountains In Sichuan Reflected the Green Water "Fig. 1", according to poetry written by Bai Juyi during Tang dynasty. He presents the landscape in Sichuan by using gravel stones. In its spatial layout, through the accumulation and dispersion, high and low, light and shade, appear people landscape in Sichuan vividly, at the same time highlight the profound artistic conception. The white space between two peaks is little, showing the right amount of space, so as to realize a kind of "far" sense of space and time. Yang tao uses thin basin to form strongly contrast between mountain, water and the basin horizontal, as a result stick out the peaks, configuration of trees and presenting a more deep and distant scenery.

\section{The Conception of "Living"}

Taoism has a devout and respectful attitude towards "live", highly praises the sanctity of life and takes life as the most important thing in life and the nature of the universe. It believes that what Taoism believes is the real world and the pursuit of the meaning and value of life, thinking that the life of human is in this living world, not the world after death ${ }^{[11]}$. "Being Immortality and becoming an immortal" is the ultimate goal of Taoism, containing a believe that they can break the rule of heaven and nature, such as the necromancer finds the longevity for the First Emperor of Qi during the pre-Qin period. Taoism attaches great importance to the continuation of life all the time, thinking that people can be an immortal by learning to be a man and accumulating good deeds just like merits and virtues. As a result breaking through the reality that life is limited. Only if people hold the life subjectivity "My fate is not governed by me not God ", can they realize the value of life and getting the spirit of eternal life beyond the people for the fear of death.

The development of bonsai had been vested the "longevity" ideology in the history. Some stones have also been given a fairy meaning of magic islands in Chinese superstition, such as Penglai, Fangzhang and Yingzhou. During the late period of Wei and Jin dynasties, social unrest caused people to bemoan the short life, and the pursuit of "living" turned to spiritual freedom, which made the intention of "living" more profound. 


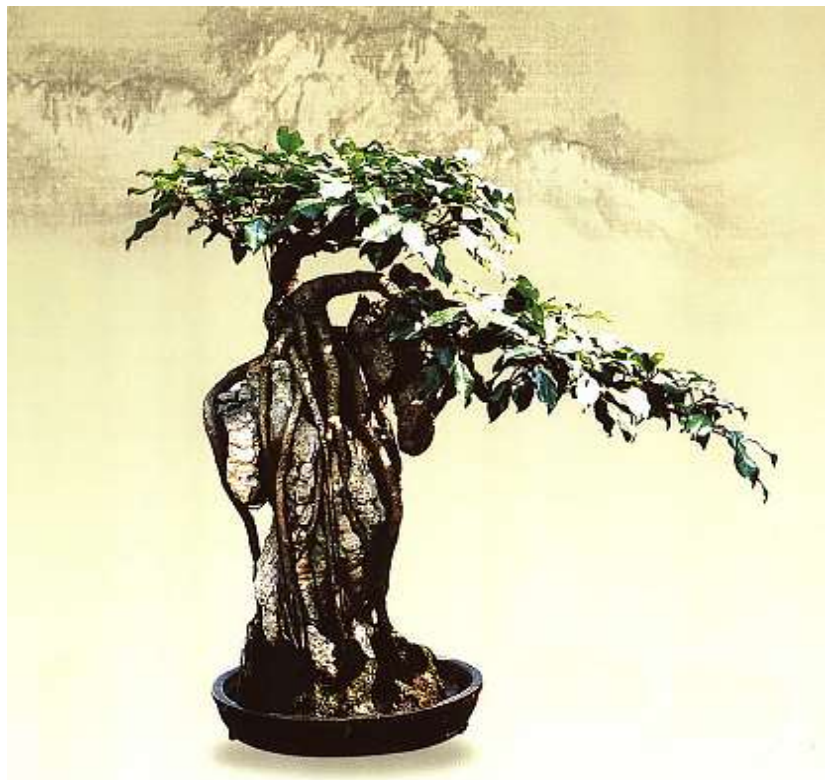

Fig. 2. The Essence of Life.

Bonsai art is a living art. The main constitution parts are plants. Its natural properties constitute the biological material basis of bonsai. The sum of various ecological factors constitutes the ecological environment of bonsai, which called The Conception of Living. When people create the bonsai, they invest much hope of life. It reflects the meaning of life existence. The idea of "living" in Taoism also affects the bonsai, the plants bonsai fuses the life of nature and getting a new lease of life, combining the cognition of lives in the universe, getting the essence and significance of "living". The Shushan cliffs are bedded for its bullheaded life, such as the following Sichuan plants bonsai made by Huang guangxin, called The Essence of Life "Fig. 2". The roots of banian are weaved, rooted in rocks and having a symbiotic relationship with stones [12]. In its branch, which is both straight and curve, the production reflects the softness and strong of life.

\section{CONCLUSION}

With more than one thousand and eight hundred years' history of evolution, Taoism has gradually formed the unique and complete system in China, which can give Sichuan bonsai great inspiration on its aesthetic. The appreciation in the article shows that the combination of them has revealed the broad and profound Chinese. If people keep trying on the fusion of aesthetics derived from Taoism with Sichuan bonsai, the prospect that Sichuan bonsai shows unique connotation and charm on the international stage will come true. As a result, the culture in Sichuan bonsai will be more vivid and lively.

\section{REFERENCES}

[1] Zhao zhilong. Heming Mountain-the Source of Taoism [J].Chinese Religions, 2008,5:48-50
[2] Deng wenxiang. Breaking Out of the Sculpture and Embracing Both Sides-A Brief View of the Development of Sichuan bonsai in the New Era [J]. Bonsai of Flowers and Trees,2016,8:10-11.

[3] Liu tao. Taoist culture [M]. Hefei: Huangshan Book Club,2014:4-8.

[4] Chen yu. Analysis on the Modeling Theory and Development Strategies of Sichuan potted landscape [J]. Science and technology information, 2009,6(8):220-222.

[5] Pan xianyi. The Aesthetic View of Landscape in Taoism[J]. Chinese Culture Forum,2014,6:101-107.

[6] He gu. Natural Rhythm Leads to High Distance - landscape Bonsai with Art of Poetry and Painting[J]. Garden,1998,6:53-53.

[7] Wang fengxiu. Artistic aesthetics of Taoism in Chinese Landscape Painting[J]. Chinese Painting and Calligraphy,2007,9:156-158.

[8] Hang zhoudong. Artistic Features in Landscape Bonsai[J]. Yunnan Forestry,2015,3:70.

[9] Fang bin. The brief analysis of Lao Tzu's aesthetic Ideologies[J]. Journal of Jiangxi University Administration

[10] Liu jinhai. Art of Bonsai and Flower Arrangement[M]. Beijing: China Agricultural Press,2008:140-142.

[11] Wang yi. Sages and Immortals: the Comparison of the Concept of Life and Death between Confucianism and Taoism [J]. Journal of Lao Tzu Studies,2014,0:189-196.

[12] Zhang zhongmin. Sichuan Bonsai Art [M]. Hefei: Anhui Science and Technology Press,2015:112-114. 